\title{
Worlds Enough, and Time: Musings on Foundations
}

\author{
Mark A. Brown
}

\begin{abstract}
Belnap's work on stit theory employs an Ockhamist theory of branching time, in which the fundamental possibilia within models are commonly taken to be moments of time, connected into a tree-like branching structure. In the semantics for alethic modal logic, necessity is characterized by quantification over relevant possible worlds within a model, yet Belnap refers to an entire model of branching time as our world, seemingly leaving no room for non-trivial quantification over worlds within a single model. This chapter explores the question how the notion of possible worlds should be understood in relation to an Ockhamist framework, in order to be able to combine an account of alethic modalities with an account of branching time and stit theory. The advantages and drawbacks of several alternative approaches are examined.
\end{abstract}

\section{Core Features of Ockhamist Branching Time}

Systems of logic based on Ockhamist models of branching time ${ }^{1}$ offer rich opportunities for the representation of concepts that are not as readily or as sensitively representable in systems based on possible worlds. There are a number of such systems based on models of branching time, suitable for interpreting a variety of formal languages, and models for different systems will differ in various ways, having different components, different constraints on their structure, and different associated satisfaction conditions.

\footnotetext{
${ }^{1}$ I distinguish these from models of branching spacetime, which have a more complex structure. Some of the remarks made here about models of branching time will have analogous application to models of branching spacetime, however.
}

M. A. Brown ( $\varangle)$

Department of Philosophy, Syracuse University, 541 Hall of Languages,

Syracuse, NY 13244, USA

e-mail: mabrown@syr.edu 
Some broad features of the semantics, however, are held in common. A branching time model $\mathcal{M}$ for a language $\mathcal{L}$ will include among its components some non-empty set $\mathbf{M}$ of moments, a binary ordering relation $<$ among the moments, and a valuation $\mathcal{V}$, assigning an extension to each atomic formula of the language and, more generally, an extension to each non-logical constant of the language, at each point of valuation in the model. ${ }^{2}$ Some structural constraints are also held in common: in particular, $<$ is constrained to be a strict partial ordering with no backwards branching:

(1) $\neg(m<m)$

$(m \in \mathbf{M})$

\section{(irreflexivity)}

(2) $m_{1}<m_{2} \& m_{2}<m_{3} \Rightarrow m_{1}<m_{3}$

$\left(m_{1}, m_{2}, m_{3} \in \mathbf{M}\right)$

\section{(transitivity)}

(3) $m_{1}<m_{3} \& m_{2}<m_{3} \Rightarrow m_{1}<m_{2} \vee m_{1}=m_{2} \vee m_{2}<m_{1}\left(m_{1}, m_{2}, m_{3} \in \mathbf{M}\right)$

\section{(no backwards branching)}

The partial ordering induces a tree structure and within any tree a maximal linearly ordered subset of moments is called a history. We shall let $\mathbf{H}$ be the set of all histories in the model. For the logic of action, other components may be added, most commonly a non-empty set $\mathbf{A}$ of agents and an associated choice function $\mathbf{C}$, assigning to each agent $a$, at each moment $m$, a partition $\mathbf{C}_{a}^{m}$ of the set $\mathbf{H}_{m}$ of all histories passing through $m$. In such cases, two further constraints are imposed:

(4) $m<m^{\prime} \in h_{1} \cap h_{2} \Rightarrow c_{1}=c_{2}$

$$
\left(c_{1}, c_{2} \in \mathbf{C}_{a}^{m} ; h_{1} \in c_{1} ; h_{2} \in c_{2}\right)
$$

\section{(no choice between undivided histories)}

(5) $a \neq b \Rightarrow c_{1} \cap c_{2} \neq \varnothing$

$$
\left(a, b \in \mathbf{A} ; c_{1} \in \mathbf{C}_{a}^{m} ; c_{2} \in \mathbf{C}_{b}^{m}\right)
$$

\section{(independence of agents)}

Pairs $m / h$ in which moment $m$ falls within history $h$ are called points of evaluation. ${ }^{3}$ The semantics for branching time systems is normally Ockhamist: formulas are evaluated at points of evaluation rather than simply at moments, with the result that a future-tense statement, in particular, may be true at a given moment relative to one of its histories into the future, but false at the same moment relative to another. As I stand here at a given moment deliberating whether to visit my mother, along some histories my mother will soon be happy because I visited her, while along others she will soon be disappointed because I didn't. There is, at this moment, no simple fact of the matter about whether she will soon be made happy or soon be disappointed.

\footnotetext{
${ }^{2}$ We shall soon examine the question just what these points of valuation should be.

3 We distinguish these, at least temporarily, from points of valuation.
} 
Some propositions will, however, be settled at a given point of evaluation: settled true if true at the given moment along each of the histories through that moment; settled false if false along each.

The Ockhamist satisfaction conditions for the most basic operators, then, are these:

$\begin{array}{lllr}\text { (SCH) } & m / h, \mathcal{M}=\mathrm{H} p & \text { iff }\left(\forall m^{*}<m\right)\left[m^{*} / h, \mathcal{M}=p\right] ; & \text { ( } \forall \text { past) } \\ \text { (SCP) } & m / h, \mathcal{M}=\mathrm{P} p & \text { iff }\left(\exists m^{*}<m\right)\left[m^{*} / h, \mathcal{M}=p\right] ; & \text { ( } \text { past) } \\ \text { (SCG) } & m / h, \mathcal{M}=\mathrm{G} p & \text { iff }\left(\forall m^{*}: m<m^{*} \in h\right)\left[m^{*} / h, \mathcal{M}=p\right] ; & (\forall \text { future) } \\ \text { (SCF) } & m / h, \mathcal{M}=\mathrm{F} p & \text { iff }\left(\exists m^{*}: m<m^{*} \in h\right)\left[m^{*} / h, \mathcal{M}=p\right] ; & (\exists \text { future) } \\ \text { (SCSett) } & m / h, \mathcal{M} \models \text { Sett } p \text { iff }\left(\forall h^{*}: m \in h^{*}\right)\left[m / h^{*}, \mathcal{M}=p\right] . & \text { (settled) }\end{array}$

In our discussion in this chapter, we will assume that our models do have, or have the functional equivalent of, a non-empty set of moments, an ordering relation obeying constraints $1-3$, and a valuation, that they have Ockhamist satisfaction conditions, and that if they also have agents and a choice function (or some functional equivalent), these obey constraints 4 and 5 above. We observe, moreover, that branching time models seem to reflect arrays of possibilities of various sorts, and consequently it is not evident that any additions to the models would be required-or even that any would be appropriate-in order to support alethic modalities.

These rather minimal assumptions leave open a number of options. There are four notably unspecified details of interest to us as we use such systems as the foundation of a logic of action and as we compare such systems with ones based on possible worlds:

(i) does it make sense to suppose distinct moments can be simultaneous?

(ii) are the points of valuation moments or are they instead moment/history pairs?

(iii) in such models, what should count as a possibility-i.e. as a possible world?

(iv) do we require that the set of moments in a model be pastwards connected?

\section{Newton Versus Einstein}

In common discourse, we expect to be able to say where we would be at this time if we had taken a different road. Einstein says that strictly speaking, we can't: that there is no such thing as absolute simultaneity. Our common parlance is based on intuitions which are more Newtonian than relativistic, and the Newtonian view includes a linear conception of time, rather than a branching one. One may wonder, however, whether the non-relativistic aspect of Newtonian physics might be separable from its assumption of temporal linearity. Is it reasonable to entertain an account of branching time which makes room for simultaneity between moments on distinct branches, and thus in this respect is Newtonian rather than relativistic?

Some systems of branching time logic include an equivalence relation $\mathbf{I}$ among moments. In such models the equivalence classes under $\mathbf{I}$ are called instants, and 
appropriate constraints are imposed to ensure that each history intersects each instant at exactly one moment, and that the ordering of moments on any history induces a linear ordering among instants that is independent of the choice of history. Such systems are undoubtedly internally consistent. The question, however, is whether they are conceptually coherent and, further, whether they are of use in a relativistic world despite their non-relativistic character.

To answer this, we may begin by noting that Newtonian mechanics remains of great utility even in a relativistic world. At normal velocities, over normal distances, for everyday purposes, relativistic effects are for the most part negligible. Using the more complex apparatus of relativity theory to solve normal engineering problems would be needlessly complex and inexcusably inefficient. Similarly, we may hold, a logic of branching time with instants may for most ordinary purposes be only negligibly inaccurate compared with a relativistic logic of spacetime, and might be rewardingly more efficient to use.

There remains, however, some doubt about whether the addition of instants to a theory of branching time actually produces benefits in proportion to the additional complexity it introduces. Thus far, instants have found application chiefly in the characterization of one operator in the logic of action, the achievement stit, or astit, operator. ${ }^{4}$ This is used to express the claim that an agent has by her action achieved the state expressed by a certain sentence $s$, and explains this as the claim that she had, at some prior moment, a choice which, exercised as it was, assured that whatever else intervened, $s$ would be true at this instant, but exercised differently might under some conditions have left $s$ false at this very instant. While the notion of an instant plays an essential role in this definition, it is also the weak point in the concept, from the point of view of applicability, since we seldom know, or care, what would have been true at this very time (even in the relativistically innocent sense we might call nominal time: when the clock there reads the same as the clock here does now) along other histories. Rather, we are likely to be concerned about what would have been true at approximately this time, or even in some cases just eventually.

When asked at 3 o'clock who shut the door, I claim that I did, some five minutes ago. In doing so, I need not claim that, given what I did, and no matter what happened between 2:55 and now, the door would have been shut when the clock struck 3 . I need only maintain that no matter what happened between the time when the clock read 2:55 and the time the clock read 3:00 the door would have been shut for a while and that, as it happens, it has remained shut until now. There was perhaps some risk that someone would open it again in the interval, but in fact nobody did. This claim makes no use of the notion of the same time in other circumstances and thus doesn't require instants. Accordingly, it appears that instants are of little practical value as a component of a system of logic of branching time.

This suggests the following strategy: to omit instants, leaving a system which, though not fully relativistic, is at least not in conflict with relativity, and in those infrequent cases in which it seems desirable to compare moments chronologically

\footnotetext{
${ }^{4}$ Here, 'stit' is an acronym for 'sees to it that', used in naming any of a variety of operators in logics of action based on branching time.
} 
across histories, to do so only by comparing the states of clocks and calendars, or other relevant indicia, at those moments.

It suggests, as well, that another temporal operator, often used in the literature on linear time, might also be kept available in the discussion of branching time, to express the notion for an interval (however brief) of time. The satisfaction condition could be given as

(SCT) $\quad m / h, \mathcal{M} \models \mathrm{T} p \quad$ iff $\quad\left(\exists m^{\prime \prime}>m\right)\left(\forall m^{\prime}: m<m^{\prime}<m^{\prime \prime}\right)\left[m^{\prime} / h, \mathcal{M} \models p\right]$

(for a time)

\section{The Enigmatic Present}

In the indeterminist philosophy which the logic of branching time is intended to capture, we consider the past settled, but the future unsettled: there is only one path back, but there are many forward. The present, however, is somewhat enigmatically situated on the cusp between these two: is it settled, like the past, or unsettled, like the future? (Often "unsettling", to be sure, but unsettled?).

The technical issue associated with this query is this: should the valuation $\mathcal{V}$ in models for branching time assign values to atomic sentences at moments or should it instead assign such values at moment/history pairs? We might call these the static and dynamic views of moments, respectively.

When we consider moments to be analogs of possible worlds, each associated with a maximal consistent set of basic facts and their consequences, we expect the atomic formulas to be true or false at a moment, even though more complex formulas involving tense operators must be evaluated at moment/history pairs. This is the view that a moment is a state through which some histories pass, and that the atomic formulas of the language are purely stative and should therefore be determinately true or false at any given state. On such a static view, the valuation should assign a truth value to each atomic formula at each moment, rather than at each point of evaluation. This has the odd result that some formulas get truth values at moments, but most only get truth values at moment/history pairs. We then have an odd contrast between points of valuation (moments) and points of evaluation (moment/history pairs), and a correspondingly odd contrast in treatment between atomic and non-atomic sentences.

If, on the other hand, we hold that formulas — all formulas — have truth values only with respect to a point of evaluation - a moment/history pair - then in the absence of further constraints the valuation is free to assign different values to the same atomic formula at the same moment, but along different histories. Then, for example, "the cat is alive" can be true at $m / h_{1}$ and yet "the cat is dead" be true at $m / h_{2}$. If the cat in question is Schroedinger's, suffering from that malaise known as quantum uncertainty, this might seem a desirable feature of the system.

Similarly, "I choose $c_{1}$ " and "I choose $c_{2}$ " could be true at the same moment- the moment of choice-but along distinct histories. On this view, then, atomic formulas do not (or perhaps do not all) express states. They are (some of them, at least) 
more dynamic than static. On this dynamic view, the present is then in some degree unsettled, just as the future is, because the present sometimes prefigures aspects of the future.

(We might entertain the thought that there really is no present-only the past and the future and the distinction between the two. In that case, however, the very existence of the present tense, ubiquitous though it is in language, would have to be considered metaphysically misleading. Moreover, and more important for our limited topic here, the notion of moments of time would lose its place in our theory, with intervals assuming the dominant role.)

By way of contrast we see that on the static view an atomic formula expressing the claim "I choose $c_{1}$ " really has no place in the language. It cannot be true at the very moment at which there is a real choice between $c_{1}$ and $c_{2}$, because then at any later point of evaluation $m / h$ in a history within choice $c_{2}$ it would be true both that I had and that I had not chosen $c_{1}$. To avoid such a contradiction we would have to hold that there are no moments at which I choose-only moments at which I have chosen.

Earlier, I said that we consider the past settled and the future unsettled. But strictly speaking, if the future is unsettled the past is as well, in the sense that not all assertions about the past will be settled true. For example if a future tense formula $F p$ is not settled (is neither settled true nor settled false) at a point of evaluation $m / h$, then the formula PF $p$ is not settled either. If $F p$ is satisfied at $m / h$ but not at $m / h^{\prime}$, for example, then PF $p$ will likewise be satisfied at $m / h$ but not at $m / h^{\prime}$. So what, then, is settled about the past? The original thought was that the facts of the pure past (uncluttered by embedded references to the future) are settled, because there is no pastwards branching to provide alternate pasts. If individual moments may be unsettled, even with respect to sentences including no overt reference to the future, then this thought might seem to fall apart. Actually, however, it is not quite as bad as that.

Suppose that, at a moment $m$, an atomic sentence $s$ is true along some histories through $m$, but false along others, and consider how things will look from the perspective of a later moment $m^{\prime}$. Various histories run through $m^{\prime}$. In order to be able to say that the past is settled, we need to be assured that at $m^{\prime}$ the truth value of a past-tense sentence such as $\mathrm{P} s$ will be the same no matter which history through $m^{\prime}$ we are considering. So let's consider two such histories, $h_{1}$ and $h_{2}$. Since $m^{\prime}$ is later than $m$, both these histories run through $m$ as well. If $s$ is true at $m / h_{1}$ but false at $m / h_{2}$, then $\mathrm{P} s$ will be true at $m^{\prime} / h_{1}$ but might be false at $m^{\prime} / h_{2}$. To avoid this risk, and save the settledness of the past, all that would be required would be to add a constraint reminiscent of the principle no choice between undivided histories, namely:

(4*) $m<m^{\prime} \in h_{1} \cap h_{2} \Rightarrow \mathcal{V}\left(s, m / h_{1}\right)=\mathcal{V}\left(s, m / h_{2}\right)$

(for $s$ atomic)

\section{(no momentary differentiation between undivided histories)}

This would still permit us to have $\mathcal{V}\left(s, m / h_{1}\right) \neq \mathcal{V}\left(s, m / h_{3}\right)$ for any history $h_{3}$ that doesn't run through $m^{\prime}$, and thus would be compatible with holding that the present can be unsettled. 
This begins to feel like a very artificial and unmotivated position, however. Perhaps the uneasiness it provokes can be seen this way: If it is possible to have $\mathcal{V}\left(s, m / h_{1}\right) \neq$ $\mathcal{V}\left(s, m / h_{3}\right)$, then it is no longer clear what it means to say that the two histories $h_{1}$ and $h_{3}$ are merged at that moment $m$ or, to put it the other way around, what it means to say that $m$ is really the same moment in both histories. The contingent truths at $m / h_{1}$ might be entirely distinct from those at $m / h_{3}$. The very idea of branching time then seems to fall apart, leaving us merely with a set of potentially unrelated histories. In short, to preserve the view of time as branching, we need to preserve the picture of moments as states, like still frames in a film, described by atomic formulas which give the basic facts, and with the dynamics of the model arising only from the connection of moments into histories, just as the dynamics of the movie arise from the sequence of the static pictures in its individual frames.

On the whole, then, it seems preferable to give up present tense atomic formulas of the special form a chooses $c$ rather than to give up our general picture of what binds histories together at a moment, and accordingly it seems to be the norm in the literature that valuations are assumed to assign truth values at a moment rather than at a moment/history pair. ${ }^{5}$

If, then, we are not to abandon altogether the thought that sentences such as "John chooses the left fork in the road" can be expressed in our formal language, we must recognize them as complex in some way_not simple, and perhaps not truly presenttense. It might seem that this would call for introducing a new operator of some sort, but in fact the well-known deliberative stit, or $d s t i t$, operator will serve perfectly well here: John deliberatively sees to it that he takes the left fork, i.e. he makes a choice which assures that he takes the left fork, while there is another choice available which would not assure this outcome. Along some histories through the present moment, this will be true, while along others it will be false, just as we might expect.

From here on, we will assume that the valuation $\mathcal{V}$ assigns values at moments, rather than at points of evaluation. Schroedinger's cat will have to fend for itself.

\section{What is a World?}

As we compare systems based on possible worlds with ones based on an Ockhamist logic of branching time, we sense that the systems based on branching time are more fine-grained and hence potentially more sensitive. But this implies that in branching time systems we should be able to do, or mimic, any of the things one could do with possible worlds. In particular, we ought to be able to introduce the alethic modalities: possibility and necessity. In possible worlds models, the possibilities are represented by the possible worlds. The question then arises: What should be considered to

\footnotetext{
${ }^{5}$ We could, of course, declare that we assign values to formulas only at moment/history pairs, but add the constraint that in the case of atomic formulas, the valuation must be the same at pairs that share the same moment. This would simply be a way of accepting the static account, while offering the surface appearance of uniformity of treatment between atomic and non-atomic formulas.
} 
represent possibilities within branching time models? Pondering this, we discover we have an embarrassment of riches.

We notice first that the moments, by virtue of their being the units of construction within branching time models, play a role analogous to that of possible worlds. Moreover if, as we've suggested should be the case, the valuation $\mathcal{V}$ assigns values to atomic formulas at moments rather than at moment/history pairs, then each moment is associated with a maximal consistent set of present-tense formulas: the atomic formulas specified by $\mathcal{V}$, together with their logical consequences. This bears some analogy with the way in which possible worlds are associated with maximal consistent sets of non-modal formulas in a typical possible worlds model for, say, S4. But the analogy is limited. Whereas in a model for $\mathbf{S 4}$ there is also a maximal consistent set of formulas, including modal formulas, associated with each world, there will be no such set associated with a moment, since in a branching time model future tense formulas, in particular, will get their truth values only at moment/history pairs, not at moments.

Since the history, not just the moment, is crucial, we might consider points of evaluation-moment/history pairs — as candidates for the role of possibilities. Each of these will be associated with its own maximal consistent set of formulas which, collectively, will fully describe a possible historical situation-past, present and (within its history) future. This matches up with the fact that in possible worlds models the points of evaluation are just the individual worlds. So far, so good, but there is an oddity here: each moment/history pair within a given history will essentially represent the same possibility as each of the others, though from the perspective of a different moment in the history. So there is really only an indexical difference between points of evaluation within the same history, a difference concerning which moment within the history is thought of as the present, while the sequence of events will be the same.

This observation suggests that we should consider whole histories as the fundamental possibilities within branching time models, each such possibility specifying one way the complete current state of the world could be at each moment throughout its history. To be sure, there will be no single maximal consistent set of formulas associated with a history, since the values of present tense formulas, for example, will change from moment to moment within a single history. But we could accept this as just another indexical phenomenon, with the values of sentences involving the term 'now' to be resolved by reference to an index, just as sentences involving the term 'me' must be. ${ }^{6}$ Given that we are permitting tensed language, we would face the same problem in possible worlds theory as well. Evaluation of indexical sentences will require that we supplement the specification of the world with a value for each of the needed indices, including a temporal index.

We now begin to have a basis for distinguishing what we might call internal and external possibilities. Within a given history perhaps the light switch is sometimes on, sometimes off. If so then that history includes the internal possibility of the light

\footnotetext{
${ }^{6}$ It won't be just present-tense sentences that will need a temporal index for their evaluation, of course: all tensed statements will be indexical relative to a history.
} 
switch's being on, as well as the internal possibility of its being off. This contrasts with the external possibilities such as that there at some point be (as in the given history) or (as in others) never be a light switch in the room at all. A contrast between internal and external possibilities, if it withstands further scrutiny, provides a basis for two layers of alethic modality, requiring two distinct sets of alethic operators. That is a prospect worth investigating to see whether it can be put to good use in some way. That investigation would take us beyond the scope of this essay, however.

Going further, we might consider whole trees to be correlates of possible worlds. In doing so, we would be acknowledging that some possible worlds are, by virtue of their branching, laden with potential for choice, and full of internal possibilities. Now we would need to treat both moment and history as indices to be specified in addition to the specification of the tree, in order to induce a value for most sentences. Indeed we might find ourselves with three layers of alethic modality based on three different world-like units of construction: moment/history pairs, histories, and trees, respectively.

We'll reflect further on this in the coming sections.

\section{Chronological Unity and Belnap's World(s)}

There remains the question whether our models should require that time be pastwards connected. The constraint in question would be this (with $\leq$ defined in terms of $<$ in the obvious way):

(6) $\left(\exists m_{0} \in \mathbf{M}\right)\left[m_{0} \leq m_{1} \& m_{0} \leq m_{2}\right]$

$\left(m_{1}, m_{2} \in \mathbf{M}\right)$

\section{(pastwards connection)}

Constraints 1-3 ensure that the moments in a model are organized into trees. Adding constraint 6 would ensure that there is only one such tree per model.

We focus first on models which are pastwards connected. One interesting observation is that if, in such models, we take the histories as correlates of possible worlds, we find that to determine whether two individuals appearing in different histories within a single model are or are not identical, we need only trace them back in time to see whether they have a common origin at some moment included in both the histories. So provided we are able to trace identity back through time, we get a natural solution to what would have been the problem of trans-world identity but which is now recast as the non-problem of trans-history identity.

But from another point of view, because the various histories in a branching time model are all connected it is reasonable to consider, as Belnap does, that the entirety of the structure in one pastwards-connected branching time model represents "our world". Such a model depicts a world rich with internal possibilities, past and present, and rich with alternative histories, each of them a possible history of the actual world, rather than an actual ${ }^{7}$ history of a different possible world.

\footnotetext{
7 Actual according to an indexical understanding of that notion, that is.
} 
From that point of view, distinct branching time models represent genuinely different ways a world might be, each with its own branching structure, its own histories, its own internal possibilities. These pastwards-connected branching time modelsBelnapian worlds, as we may call them ${ }^{8}$ — will be in some respects much like the possibilities represented by the Kripkean worlds of normal systems of modal logic. They will differ from such worlds in at least two respects, however. First, of course, each Belnapian world has a rich internal structure which Kripkean worlds lack. But also, these Belnapian worlds are isolated from one another in separate models, whereas Kripkean worlds coexist within the same model. Accordingly, we might recognize the possibilities internal to a Belnapian world as real possibilities (relative to that world) and recognize the possibilities represented by the availability of other models as merely nominal possibilities - other ways the language might have been given application. We will examine this thought again in Sect. 7.

At this level, the problem of trans-world identity might be thought to surface again, but our having noted that there is no special problem of trans-history identity within a branching time structure can be the occasion for reconsidering the role different worlds play. If different Belnapian worlds only appear in different models, i.e. if we impose on models the requirement of historical connection, then perhaps we can profit from some reflection on the general nature of formal models, examining the comparative role of different models, and therefore of different Belnapian worlds.

So at the risk of being pedantic, let us review the basic features of models.

\section{The General Character of Models}

First of all, we note that a model for a logical system is always a model with respect to a language, and that this language is held fixed for the whole class of models for a given system.

Second, we note that each model will have two distinguishable components-a structure and an interpretive scheme. The structure is our stand-in for the world; the interpretive scheme links the language to the structure and in doing so represents the way the language is understood to connect with the world. The structure in turn has two sub-components: an ontology, which varies from model to model, and a set of structural constraints which remain constant across models. The interpretive scheme also has two sub-components: a valuation, which varies from model to model, and a set of satisfaction conditions which is invariant across models.

The ontology gives the array of types of entities that are taken to be explanatorily fundamental, for the level and style of explanation undertaken by the system. All other types of entities acknowledged by the system are constructed from, or

\footnotetext{
${ }^{8}$ We may, but Belnap may not approve. There is, after all, only one world, our world, and the real possibilities are all included within it. However the alternative worlds we consider here are logically possible - logically consistent - , and even metaphysically possible: consistent with the metaphysical commitments built into our definition of models and our satisfaction conditions.
} 
possibly in some cases supervenient on, this ontological base. Typically, for Belnapian worlds the ontology may include such kinds of items as moments, agents, acts, and perhaps instants. Fundamental relations and functions built into models- the relation of temporal precedence among moments or a choice function for agents, for example-might be construed either as part of the ontology or as contributing to the structural constraints, but generally can be and are treated as part of the ontology. There is a relation < between moments; that sounds like ontology. The relation is transitive; that's structural.

It is important to note that in the general specification of the class of models for a system the ontology is not normally constrained to include any specific entities, i.e. does not include any distinguished objects. The definition of models may specify that there are to be a number of agents in each, but it will not normally identify any of them; it will not normally specify that I am to be among them, for example, nor that any other specific entity is. On the other hand, it is equally important to note that any given model in the accepted class of models will have a specific ontology, filled with specific entities. Model 1 may have a set $\mathbf{A}_{1}$ of agents, for example, and model 2 a set $\mathbf{A}_{2}$. But nothing is said, normally, about whether sets $\mathbf{A}_{1}$ and $\mathbf{A}_{2}$ share any elements. Similarly with moments and other types of entities. In general, then, two models of the class may share some items in their ontology or they may not. Normally there are no constraints on models that will rule out either of these alternatives. This is one aspect ${ }^{9}$ of what we might call our metaphysical diffidence: we restrain ourselves from pretending to present all the details of the constitution of reality.

The structural constraints represent those fundamental assumptions about the nature of the universe that go beyond answering the question what kinds of things there are, to answer questions about how the entities of the universe are organized in relation to one another. In Belnapian worlds these constraints include such constraints as that time does not branch pastwards and, even more fundamentally, that the relation of temporal precedence is irreflexive and transitive. I tend to think of these constraints as metaphysical commitments, though the distinction between metaphysics and physics might blur here. Taken together, however, the class of models satisfying the structural constraints reflects the metaphysical foundations taken as underpinning the language.

The valuation assigns to each non-logical atomic component of the language an extension of an appropriate type in, or constructible from, the ontology. Finally, the satisfaction conditions exploit this assignment to make it possible to calculate truth values for sentences of the language at each point of evaluation, and in doing so constrain the meanings of the logical constants of the language.

Given this general understanding of the nature and role of models, a little reflection indicates that different models represent different logical possibilities, each internally consistent and each consistent with the metaphysical foundations reflected in the ontology and structure that defines the class of models. The necessity which can be defined via such models will be of just one sort: logical necessity relative to the

${ }^{9}$ The most fundamental reflection of our metaphysical diffidence is the fact that we entertain a whole class of models, and do not designate one of these as the real model. 
constraints. The necessary truths are the logically necessary consequences of the acceptance of the satisfaction conditions and the acceptance or the imposition of those constraints.

\section{Comparing Belnapian Worlds}

When we compare two Belnapian worlds, they will be alike in the broad outline of their ontology and their metaphysics. But although they will agree on what kinds of entities are available for discussion, they will commonly differ concerning which particular entities of a given kind are involved. We may require that each model have a non-empty set of agents, for example, but we don't presume to specify what set, and as a reflection of this metaphysical diffidence different models needn't have the same set. On the other hand, they needn't have distinct sets, either.

Because each model in a given system will be a model for the same language, the models will agree about what names are available to be given to items in the ontology, but will typically not agree about which objects bear which names. So if 'Belnap' appears in the lexicon of the language, and is constrained by its lexical category to name an agent, it may name our favorite logician in one model closely corresponding to the actual world, but in another model might name some seventeenth-century nun who developed, say, an irrelevance logic. Names that are assigned distinct denotations in one model might be two names for a single entity in another. So although we can trace names from world to world, we cannot use those names to trace entities outside the boundaries of a single world. We can say that the name 'Belnap' is used differently in different worlds, but we cannot on that basis say that Belnap himselfour Belnap-even occurs in those worlds, much less that he has different properties. On the other hand, Belnap himself will occur in some of those worlds, though it is anybody's guess what name(s) the language assigns him there.

One consequence of all this is that a form of the problem of trans-world identity does arise across the class of Belnapian worlds, even though there is little problem of trans-history identity within a given Belnapian world. Another consequence seems to be that it makes little sense to even contemplate judging specific causal connections using alternative Belnapian worlds. In both these cases, however, the problem may be less compelling than it seems at first. Let us look more closely.

It is true that the agent called Belnap in one Belnapian world may bear no relation to the agent so called in another, and that therefore if in one Belnapian world the sentence 'Belnap is a seventeenth century nun' is true, that does not by itself establish the possibility that Belnap could have been a seventeenth century nun. But if we contemplate the full array of all Belnapian worlds, we will find many in which Belnap himself does appear, and among those many in which he is accorded the name 'Belnap'. Moreover, among these, there is (barring special restrictions on the class of models) at least one identical in all internal details to the one in which 'Belnap is a seventeenth century nun' is true, except that the object there named 'Belnap' really is our real Belnap, not merely some name-sake, and so is the basis 
for saying, within any Belnapian world, that it is possible that Belnap could have been a seventeenth century nun. We may not be able to trace identity from world to world, but the richness of the array of Belnapian worlds renders that limitation of no practical consequence for the evaluation of sentences about possibility and necessity. The fact that we cannot be sure that this Belnapian world has the Belnap we are talking about doesn't matter: there will be another Belnapian world descriptively just like it that does, and that uses the same name for him. It's possibilities are therefore his possibilities. Similar remarks will apply to tracing causal connections: connections that can be traced by name will also be traceable through cases (and there will be such cases) in which the name is applied to the same object.

But in what sense of 'possibility' can possibility be attributed here? Normally, in Kripkean systems, we count only worlds within the same model as providing a basis for claims of possibility. We don't acknowledge worlds from other models as relevant. At best, they provide for the logical possibility of the truth of certain claims, an acknowledgement that the language could have been used that way without contradiction, and without violating the metaphysical assumptions which underly it, whether it is in fact applied that way or not. So to the extent that we see Belnapian worlds as isolated from one another in separate models, we make them irrelevant to one another for alethic purposes, determining real possibility, though they remain relevant for purposes of determining logical possibility, validity and logical truth.

Suppose, now, that we nonetheless contemplate setting Belnapian worlds to some of the tasks normally assigned to Kripkean worlds, such as making it possible to introduce alethic possibilitation and necessitation operators into the language. In Kripkean systems, the ontology includes possible worlds, and various such worlds will be gathered together into a single simple Kripke model for, say, the system S5. So we typically have many worlds in a Kripkean model for S5, and understand a sentence $p$ to be necessarily true at world $w$ in the model iff $p$ itself is true at each world in the model. Here, incidentally, our metaphysical diffidence manifests itself again in the fact that we do not presume to specify how many, nor which, possible worlds there are, and we permit (nay, require) the array of different models to offer a full array of different answers to such questions.

Introducing explicit possibilitation and necessitation operators $\diamond$ and $\square$ into the language for $\mathbf{S 5}$ enables us to create formulas $\diamond p$ and $\square p$ to express the claims that the claim expressed by $p$ is possibly true or necessarily true, respectively. Now if we try to put Belnapian worlds into an otherwise Kripkean model where there would previously have been Kripkean worlds, we get a sort of supermodel whose ontology includes Belnapian worlds which are themselves Ockhamist models, each with their own internal ontology. Can we make any sense at all of this? Let's think it through.

First, we will continue to have a single common language for each of the Belnapian worlds in the supermodel, and indeed across the various supermodels appropriate to our system. The language will, presumably, be the language we would have used for the Belnapian worlds themselves, but augmented by the new alethic operators. Since at the moment we're thinking only of what we might call a Belnapian S5, 
no additional structural constraints seem needed. ${ }^{10}$ However, when we turn to the valuation and the satisfaction conditions, things begin to look a bit more complex. The first question we face is: what are we to take as the points of evaluation in a supermodel? The analogy with Kripkean models for $\mathbf{S 5}$ would make each Belnapian world a point of evaluation, while the analogy with Belnapian models would make moment/history pairs within Belnapian worlds the points of evaluation.

What at first blush seems a reasonable hybrid, namely to accept moment/history/ world triples $\mathrm{m} / \mathrm{h} / \mathrm{w}$ as points of evaluation might turn out not to be a hybrid at all. This depends on how we view the sets of moments in distinct Belnapian worlds. If we assume (or require) that no moment occurs in more than one world then a given moment/history pair can only occur in a single Belnapian world. Then we are effectively back to just moment/history pairs, since settling on values for $m$ and $h$ will force a value for $w$; then the satisfaction conditions for $\square p$ at a given point of evaluation in the supermodel will naturally be simply that $p$ be true at each point of evaluation in the supermodel. No obvious problem here. As in S5, it will be automatic that whenever a formula $p$ is valid, the corresponding formula $\square p$ will also be valid. However if $p$ is not valid, the formula $\square p$ can be true in one supermodel while remaining untrue in another-really necessary, as far as that world is concerned, but not logically necessary.

That's if we assume that distinct worlds have disjoint sets of moments. Our metaphysical diffidence would suggest, however, that we might wish to restrain ourselves from such an assumption. Indeed, despite our misgivings about identifying times across separated histories within a Belnapian world, it doesn't initially seem absurd or unnatural to speak about the same time in different worlds. For linear time, this could be made to work out very simply, with each world using the same moments as every other, and with the same ordering in each world. However with branching time, much of the point would be lost if we supposed the trees in different worlds were all isomorphic to one another. And if they are not isomorphic to one another, then it is hard to see how the very same moment that occurs in one could occur in another in any meaningful way, i.e. in any way that made use of that identity. A fortiori, the same is true for moment/history pairs. Accordingly, let us overcome this bit of our metaphysical diffidence and assume that in supermodels distinct Belnapian worlds have disjoint sets of moments, and thus that a given point of evaluation in the supermodel will occur in exactly one world. ${ }^{11}$

When we look at the possibility of using Belnapian worlds in a supermodel to support other normal alethic modal operators, as in system $\mathbf{K}$, or $\mathbf{S 4}$, or $\mathbf{S 4 . 3}$, for example, the direct analogy calls for us to complicate the supermodels with a relevance relation between Belnapian worlds, and (for any except the weakest normal system, $\mathbf{K}$ ) add constraints on this relation. It would also, I would submit, be important to provide an interpretation of the relevance relation, and to justify any constraints on that

\footnotetext{
${ }^{10}$ We'll consider normal systems other than $\mathbf{S 5}$ in a moment.

${ }^{11}$ This would not rule out the possibility that moments from different worlds, though distinct, might be comparable with respect to the truth values of certain canonical forms of chronological sentences about, say, clocks and calendars.
} 
relation in terms of this interpretation. Unless we were engaging in a purely technical investigation, we should have some story to tell about what makes one Belnapian world relevant to another-some account of what one world would have to be like in order to be relevant to another. ${ }^{12}$

However, given that (as we are now assuming) each point of evaluation will occur in only one world, we could consider a relevance relation directly between points of evaluation. In the closest analogy to standard models, instead of having world $w$ relevant to world $w^{\prime}$, we could have all $w$ 's points of evaluation relevant to each of $w$ 's. Then $\square p$ would be true at a given point of evaluation $m / h$ iff $p$ is true at each point $m^{\prime} / h^{\prime}$ relevant to $m / h$. Once we contemplate such point-to-point relevance, however, we should at least consider the possibility that relevance could be more selectively defined, so that perhaps only some of $w$ 's points of evaluation would be relevant to ones in $w^{\prime}$, and perhaps only to selected points of evaluation in $w^{\prime}$. This would call for rethinking the relevance relation, to provide an interpretation which could reasonably be understood to be so selective. Depending on what that interpretation might be, we might also contemplate the possibility that the relevance relation could hold between selected pairs of points of evaluation within the same Belnapian world. In principle, these relaxations of the relevance relation open up a whole new dimension of potential sensitivity for systems based on such supermodels - a dimension surely worthy of at least preliminary technical exploration. We shall not explore it further here, however.

Looking in a different direction: instead of seeking to pursue the analogy with standard models, we could consider pursuing an analogy with neighborhood models based on possible worlds. In classical models the relevance relation relates a world to relevant neighborhoods, i.e. sets, of worlds. One common rationale for doing so is to take advantage of the fact that in possible worlds semantics, any given proposition will naturally be associated with a uniquely determined set of worlds: the worlds at which the proposition is true. The neighborhood is then used to represent the comprehensive proposition which captures all that is true throughout the neighborhood but which is false at all other worlds. Other interpretations similarly associate sets of worlds with events, or with actions. In each such case, worlds are gathered into neighborhoods in their capacity as points of evaluation, and so the apt analog for our supermodels would be neighborhoods made up of moment/history pairs, rather than of worlds. The default view would be that the neighborhoods could, and typically would, include points of evaluation from different worlds: the proposition that $p$, for example, would be represented in the supermodel by the set of all points $m / h$ at which $p$ was true. ${ }^{13}$

\footnotetext{
12 One classic illustration is the specification, in standard deontic logic, that world $w^{\prime}$ is to be considered deontically relevant to world $w$ iff $w^{\prime}$ is normatively ideal by the ethical standards in force at $w$, i.e. iff those standards are all actually met at $w^{\prime}$.

${ }^{13}$ Assuming that propositions transcend the given language, so that there can be true propositions not expressible in the language, the neighborhood consisting of all points at which $p$ is true is likely to represent a stronger proposition than simply the proposition that $p$. Nonetheless, this neighborhood is the best we can do by way of representing the proposition that $p$ short of having a completely expressive language. Even with an expressively complete language as our syntax, neighborhoods
} 
Another way in which neighborhoods have been put to use is in Lewis's logic of counterfactuals. There, given any world $w$, the worlds of a model are gathered into concentric neighborhoods relevant to $w$, with the interpretation that worlds in a neighborhood are more similar to $w$ than are worlds outside it. A counterfactual conditional $p \square \longrightarrow q$ is then vacuously true at $w$ if there is no world in any neighborhood relevant to $w$ at which $p$ is true; otherwise $p \square \rightarrow q$ is true at $w$ iff there is a neighborhood of $w$ throughout which $p \rightarrow q$ is true and within which there are worlds at which $p$ is true.

In a system using supermodels, there will be a basis for more than one sort of counterfactual conditionals, falling into two broad categories which we might call external and internal counterfactuals. External counterfactuals will, like Lewis's, involve comparing one Belnapian world with others with respect to some measure of similarity, and will be saddled with all the problems of explaining that notion of similarity. Internal counterfactuals will instead compare one history or one point of evaluation with others within a single Belnapian world.

For the internal counterfactuals it will be possible to draw on a natural sense of similarity, based on the distance back in time one must go to find a moment common to the two histories. Histories which have split off from one another only recently will in one natural sense be more similar than ones which diverged at some still earlier moment. It seems plausible to suppose that many counterfactual conditionals that occur in ordinary reasoning are best considered as internal rather than external counterfactuals, and therefore are correspondingly more intelligible than they might otherwise appear to be.

One kind of internal counterfactual can be based on a particular agent's choices. For a sentence like

\section{If John had gone left at the fork in the road, he would have come to Paris}

we could expect this to be true at $m / h$ iff at some earlier point $m_{0} / h$ one of John's choices included histories in each of which he takes the left fork, and at all points $m^{\prime} / h$ with $m_{0} \leq m^{\prime}<m$ at which John's choices include choosing the left fork, then in each of the histories in which he takes that choice he subsequently comes to Paris.

Other counterfactuals tacitly involve agents' choices, but not for a specific agent, and so do not involve specific choices. So to evaluate a sentence like

\section{If Cheney had not been Vice President, the U.S. wouldn't have invaded Iraq}

we could look for earlier branch points at which there are histories in which Cheney does not become Vice President, and verify that the U.S. does not subsequently invade Iraq in any of the most recently diverging such histories.

\footnotetext{
(Footnote 13 continued)

would represent propositions only up to logical equivalence. These subtleties, though important, do not normally deter us from considering neighborhood semantics useful, however.
} 
There remains the possibility that for some examples, as with external counterfactuals, the relevant degree of similarity between histories within a tree might take some other form than simple distance in time back to their nearest common moment. One notorious example which appears to be of this type arises if I accidentally leave my coat behind in the cloakroom at the close of a conference session, and return the next day to find it still there. ${ }^{14}$ Knowing that there were dubious characters in the neighborhood when I left, and that many individuals had access to that cloakroom during my absence, I would reject as false the sentence:

If my coat had been stolen, it would have been the most recent person to visit the cloakroom who would have stolen it.

No doubt the first really dubious character who came by after I left was likely to take my coat, pre-empting any opportunity that the most recent nefarious visitor might have had.

For such an example, it is difficult to say what the relevant sense of similarity between histories might be, but it seems clear nonetheless that no comparison with histories from other worlds is particularly apt.

For external counterfactuals, we have a choice: we could base our account on a similarity relation between worlds or on a similarity relation between points of evaluation. For a sentence such as

\section{If the match were struck, it would light}

it might be most appropriate to compare moment/history pairs (without regard to what world they were in) and focus on the ones whose factual conditions were most similar to those at the point of evaluation. On the other hand, for Lewis's example

\section{If kangaroos didn't have tails, they would fall over backwards}

it might be best to refer to similar worlds, particularly since there are probably no suitably similar moment/history pairs in our world at which kangaroos lack tails, and it would take a very different world to include such situations in a coherent way.

The moral of all this rumination about supermodels is that they open up considerable new prospects for exploration and exploitation. We now begin to glimpse the plausibility of supposing, for example, that different kinds of counterfactuals call for different accounts, and we see that supermodels might provide an environment friendly to such fine-grained discriminations. Moreover, it is not unreasonable to suppose something similar might be the case with accounts of causation, particularly if we suppose that counterfactuals play a major role in, or are in some other way closely connected with, an account of causation.

\footnotetext{
14 Ironically, this actually happened to me at the conference at which I first heard mention of this type of example.
} 


\section{Belnapian Multi-Worlds}

So far, we have been considering the uses to which Belnapian worlds might be put. Belnapian worlds involve the constraint of pastwards connection. Now, however, let us consider the consequences of relinquishing that requirement of pastwards connection. With this constraint gone, we get models within which there may be multiple trees of moments, unconnected to one another. Because each independent tree within such a model will be in many respects very like a Belnapian world, let us call such models Belnapian multi-worlds. ${ }^{15}$

A Belnapian multi-world will not be just like an arbitrary set of Belnapian worlds, because the multi-world will have a single specification of its entities, and a single valuation assigning names to those entities, rather than having separate specifications of these for each tree. Thus a Belnapian multi-world is like a coordinated set of Belnapian worlds-worlds coordinated with respect to their ontology and their assignment of names-, but we must still wonder, as in standard models of alethic logic, whether it makes sense to suppose the same entity can occur in more than one world, i.e. in more than one tree.

There is no need to assume that no moment occurs in distinct worlds within a multiworld, because the constraints on the ordering relation $<$ will assure us of this. This is another respect in which a Belnapian multi-world differs from a supermodel or a mere set of Belnapian worlds, since although it seemed overwhelmingly appropriate to assume that a given moment could not occur in distinct Belnapian worlds within a supermodel, we did have to treat this as an assumption.

If the choice function works, as usual, to assign a set of choices to each agent in the model at each moment in the model, it would appear at first that each agent is presumptively active in each of the trees - each of the worlds - in a given multi-world. But further reflection suggests that this need not be so, if (as seems unavoidable, if we are to be realistic) the "choice" given an agent at certain moments is the "Hobson's choice" consisting of just one alternative: the set of all histories through that moment. Surely this is the sort of "choice" the agent has at moments when unconscious, for example. Moreover, we might want to assign this trivial choice to agents at all moments before their birth and all moments after their death. Doing so would provide a convenient way of representing the finitude of the lives of agents while allowing a sense of their existence, and therefore their availability for reference, outside their lifespan. In 'Socrates was Greek', the name 'Socrates' will continue to have a referent even after that philosopher is no longer alive.

If the choice function can assign the trivial choice at some moments, then there is nothing to prevent its assigning trivial choices to a given agent at every moment throughout a given tree, thus effectively excluding that agent from participation in that world. As a result it is possible for worlds within a multi-world to have effectively disjoint sets of agents. ${ }^{16}$ On the other hand, of course, it is possible for such worlds

\footnotetext{
15 Belnapian worlds will, of course, be special cases of Belnapian multi-worlds.

16 Indeed, this has nothing special to do with Belnapian multi-worlds. In any Belnapian world, unless we introduce a constraint not normally imposed, it is possible for a given agent to be present in
} 
to share an active agent, and if they do, the agent will bear the same name(s) in each world within the model.

A system based on Belnapian multi-worlds would seem to provide a natural basis for an alethic necessitation operator: $\square p$ will be true at $m / h$ in a multi-world iff $p$ is true at each point of evaluation in the model. If no further complications are added, this will automatically be an S5 sense of necessity. Logical truths will, of course, be necessary truths, on this reading, but as usual the converse will not hold in general: $\square p$ may be true at each point of evaluation in one multi-world model, but fail throughout another.

It might appear that there should also be room for what we might call situationally necessary truths_claims $p$ which are necessarily true at some points of evaluation in a multi-world, but not at others. Indeed, we will have something a little like this: true claims about the past will be settled true, but might not be true at points of evaluation on other histories; and some claims about the future will be settled true at some sufficiently late points along a given history, but might not be settled true at earlier ones. But of course such cases are handled by the settled true operator Sett, and need not be considered cases of true necessity: $\square p$ will express a stronger claim than Sett $p$.

If a suitable rationale can be found for doing so, it would be technically possible to add a relevance relation between points of evaluation, with suitable constraints on this relation, so as to have the necessitation operator be an $\mathbf{S 4}$ operator, or some other normal necessitation operator.

Since there are typically multiple worlds in a multi-world model, we again have room for various types of counterfactual operators, both internal and external. The internal operators would be just like the ones in supermodels or in single Belnapian worlds. The external counterfactual operators available within a multi-model would depend on a relation of similarity among the worlds contained within that multiworld. We must not forget that there will be more than one model—more than one Belnapian multi-world. We could contemplate assembling super-multi-worlds within each of which we gather a set of multi-worlds, but the motivation for contemplating super-multi-worlds seems weak: we already have, as in standard possible worlds models, room for more than one world per model, and so don't need to gather multiworlds into super-multi-worlds to get worlds collected together.

\section{The Making of an Agent}

Typical propositional systems of the logic of action, constructed along Belnapian lines, focus on agency and the truth conditions for agentive sentences, rather than

(Footnote 16 continued)

the ontology, but not active at any moment in any history, and thus to be for all practical purposes non-existent. It's a bit difficult to see how to interpret such a situation. This perhaps argues for the introduction of such a constraint, particularly for the usual systems whose models are single Belnapian worlds. It is also possible that an agent should be active only in some histories and not in others, e.g. that there might be some histories in which the agent was, and others in which she was not, born. 
on the agents themselves. A non-empty set of agents is postulated, and the choice function indicates what choices each will have at each juncture in branching time. But little else is normally said about the nature of the agents and about what distinguishes one agent from another other than brute non-identity.

Belnap has remarked ${ }^{17}$ that in a branching spacetime system, it is possible to associate each agent with a unique set of point events, the set of those at which the agent is present. This is made possible by the fact that distinct agents cannot occupy the same place at the same time. Unfortunately, if we look merely at branching time, with no basis for discussion of spatial dimensions, no such simple account of the identity of agents is possible.

However, the usual constraints imposed on the choice function, including in particular the constraint independence of agents, do make it possible for us to look at agents in new ways. In particular, we can give some formal substance to, and gain some new insight into, the view that an agent is the sum of the choices she makes and thus that an agent is a work in progress, existentially shaping her character and her very identity through her choices.

In a Belnapian world, the constraint independence of agents assures that, strictly speaking, no two agents will be presented with the same choices at a given moment in time. Of course at the restaurant both may be choosing between having the scallops and having the mussels, but that is only to say that the choices facing one may be descriptively like those facing the other. For one thing, even if they both choose the scallops, for example, they will not get the same scallops. But more significantly, agent $a$ is choosing what $a$ will order (and, presumably, eat), not what agent $b$ will order. So even confining attention to a single moment, agents are normally ${ }^{18}$ differentiated from one another by the choices they face.

If we shift attention to the choices agents make, not just the choices they face, this becomes even clearer: even at a single moment, provided only that the agents are active, they are differentiated by their activity, which is to say: by the choices they make.

Widening our perspective to scan a history within which a moment falls, we find the agent making a succession of choices which cumulatively help define that very history, setting it apart, choice by choice, from others that had been available. The totality of those choices will be absolutely unique to a given agent, no matter whether we focus on the menu of choices the agent faces or on the choices the agent selects from that menu. Seeing this accumulation of choices along the history as uniquely associated with one agent, we can begin to consider it as constituting that agent, in which case as we survey the history we now get a strong sense of what it might mean to say that the agent is creating herself by her choices. In a different history, pursued by making different choices, she would have become a different person.

\footnotetext{
${ }^{17}$ At the $\triangle \mathrm{EON}$ '10 Conference, Fiesole, Italy, 2010.

18 There is one kind of exception to this generalization: at a given moment two agents might be given exactly the same choice by being given no choice at all. If both are asleep, for example, the choice function will presumably assign the whole of $\mathbf{H}_{m}$ as the one "Hobson's choice" available to each at moment $m$.
} 
Each agent will correspond to a unique set of partitions of histories at choice points, and along any history, there will be a particular set of choices the agent makes and without which the agent's life would not have followed that history. So from the point of view of that history, it will seem that the agent's choices will have accumulated to make the agent the individual she has become. From this point of view, a simple version of existentialism seems vindicated.

But it is more complex than that, because the choices of others also influence the history one takes, and therefore the subsequent choices with which one is faced. In the end, then, the individual one becomes is profoundly influenced by the choices of other agents, as well as by her own, and this is where the simple existentialist picture fails.

And there is another layer of complexity added when we look beyond a single history. The totality of the agent's choices throughout the Belnapian world is also uniquely associated with that agent, and reflective not merely of what the agent does become (along this history or that) but also of the agent's potential, which we may consider is equally essential to their identity. At this scale we begin to see the agent as a unique collection of possibilities for action.

When we survey this larger picture, balancing the agent's tree of possibilities against the developments of those possibilities along a given history, we find a new perspective on the old nature/nurture debate: we need not choose between nature and nurture as the sources of one's character, and we must indeed add a third factor-will. Nature has its role in providing our potential as seen in the treewide totality of the choices available to us; will has its role through our choices; nurture has its role in the choices of others which influence and limit our choices. Together they work, along any given history, to create a uniquely matured version of the agent, different from what they could have become had they not had that potential, different from what they would have become had they made different choices, and different from what they would have become had others chosen differently, as well.

Another aspect of the focus on agency rather than on agents, as we have begun to see, is that there is nothing in the models for such systems that rules out the possibility that a given agent has been making choices throughout time, and will continue to make choices throughout time along each history. This is, of course out of keeping with the fact that the logic is intended to reflect the situation of mortal agents and agents who are not perpetually active.

If we undertake the task of constructing a quantified logic of action we can expect to be able to remedy this situation, perhaps by introducing a distinguished predicate is alive into the language and making appropriate provisions for its interpretation in our models. The many quandaries associated with quantified alethic modal logic tend to make us shy away from a quantified logic of action (which could hardly present fewer such challenges) at least for the moment. But let us contemplate a slightly enhanced propositional logic of action and use this to get at least a preliminary look at some of the challenges involved in taking account of the finitude of agents.

Suppose that in addition to the other more or less standard components of models for our logic of action we include a function $\mathbf{Q}$ (for quick, in the sense of alive) 
from moments to subsets of the set of agents, with the intended interpretation that for each moment $m, \mathbf{Q}(m)$ will be the set of agents alive at $m$. To make this work in the intended way, we would need to add some constraints. One constraint would express the continuity of life for agents:

(7) if $m_{1}<m_{2}<m_{3}$ then $\mathbf{Q}\left(m_{1}\right) \cap \mathbf{Q}\left(m_{3}\right) \subseteq \mathbf{Q}\left(m_{2}\right)$

\section{(continuity of life)}

Another might express the principle that dead agents don't choose:

(8) if $\alpha \notin \mathbf{Q}(m)$ then $\mathbf{H}_{m} \in \mathbf{C}_{a}^{m}$

\section{(dead agents don't choose)}

This would assure that only a live agent ever has more than one choice, which is to say a dead (or an unborn) agent has no active influence on the course of affairs.

Note that nothing here requires that live agents have non-trivial choices at any given moment. An agent may very well be asleep, or simply inactive, at a given moment in her life.

We might also contemplate some further constraints:

(9) if $\alpha \in \mathbf{Q}\left(m_{1}\right) \cap \mathbf{Q}\left(m_{2}\right)$ then $\left(\exists m_{0}: m_{0} \leq m_{1} \& m_{0} \leq m_{2}\right)\left[\alpha \in \mathbf{Q}\left(m_{0}\right)\right]$

\section{(uniqueness of origins)}

This would assure that the same agent isn't born independently in two different histories.

(10) if $\alpha \in \mathbf{Q}(m)$ then $\left(\exists m_{1}, m_{2}: m_{1} \leq m \leq m_{2}\right)\left[\alpha \notin \mathbf{Q}\left(m_{1}\right) \& \alpha \notin \mathbf{Q}\left(m_{2}\right)\right]$

(mortality)

This assures that no agent has been alive from all time, and that none lives forever.

Such refinements of our models would probably not have profound effects on the core logic of action, i.e. on the class of valid formulas involving only the action operator, and from that limited point of view they are probably not very important. Their value lies chiefly in their ability to reflect a little more fully the underlying picture which brings us to the logic of branching time in the first place, and in opening the prospect of additional operators which would enrich our formal language in interesting ways.

Perhaps the most conspicuous such possibilities arise when a logic of action based on branching time is pressed into service in a system of logic of ability or a system of deontic logic. In a logic of ability it will certainly commonly-but perhaps not always and in every sense of ability - be a condition of ability that one be alive. To be able to drive a car requires that one be alive. To be able to evoke happy memories perhaps does not.

In deontic logic, it will be important to note that, for example, death frees one from many obligations. If I am dead, I can have no obligation to visit my father on his next birthday. Similarly if he is dead. Developing deontic logic to the point that it is able to deal sensitively with questions about abortion, homicide, accidental death, etc., could all be expected to be aided by this sort of enrichment of our models. 


\section{Conclusion}

The basic framework of the logic of branching time, and of the logic of action based on branching time offers rich opportunities for refinement and elaboration in a variety of dimensions, and in ways that deserve exploration. There is prospect for new insights into alethic modal logic, the logic of counterfactuals, and deontic logic, to cite only a few areas. Such opportunities deserve at least preliminary exploration, but such investigations will have to be reserved to another time.

The works provided in the bibliography contain material which forms the background for the discussions undertaken here.

Open Access This chapter is distributed under the terms of the Creative Commons Attribution Noncommercial License, which permits any noncommercial use, distribution, and reproduction in any medium, provided the original author(s) and source are credited.

\section{Bibliography}

Belnap, Nuel. 1991. Backwards and forwards in the modal logic of agency. Philosophy and Phenomenological Research 51: 777-807.

Belnap, Nuel. 1991. Before refraining: Concepts for agency. Erkenntnis 34: 137-169.

Belnap, Nuel, and Michael Perloff. 1988. Seeing to it that: A canonical form for agentives. Theoria 54: $175-199$.

Belnap, Nuel, Michael Perloff and Ming Xu. 2001. Facing the Future. Oxford: Oxford University Press.

Chellas, Brian F. 1980. Modal Logic: An Introduction. Cambridge: Cambridge University Press. Horty, John F. 2001. Agency and deontic logic. Oxford: Oxford University Press.

Horty, John F., and Nuel Belnap. 1995. The deliberative stit: A study in action, omission, ability and obligation. Journal of Philosophical Logic 24: 583-644.

Lewis, David K. 1973. Counterfactuals. Cambridge: Harvard University Press.

Thomason, Richmond. 1981. Deontic logic as founded on tense logic. In New studies in deontic logic: Norms, actions, and the foundations of ethics, ed. Hilpinen, Risto, 165-176. Dordrecht: D. Reidel Publishing Company.

Xu, Ming. 1997. Causation in branching time (I): Transitions, events, and causes. Synthese 112: $137-192$. 